\title{
HA Fillers: A New "Tool" to Face Facial Post Surgical Deformities?
}

\author{
Raffaele Rauso* \\ Maxillo-Facial Surgery Department, University of Campania "Luigi Vanvitelli", Italy
}

*Corresponding author: Rauso R, Maxillo-Facial Surgery Department, University of Campania "Luigi Vanvitelli”, Piazza Miraglia 80138 Naples, Italy, E-mail: raffaele.rauso@unicampania.it

Received: 13 Jan, 2020 | Accepted: 25 Jan, 2020 | Published: 31 Jan, 2020

Citation: Rauso R (2020) HA Fillers: A New “Tool” to Face Facial Post Surgical Deformities? J Clin Cosmet Dermatol 4(1): dx.doi.org/10.16966/25762826.146

Copyright: (c) 2020 Rauso R. This is an open-access article distributed under the terms of the Creative Commons Attribution License, which permits unrestricted use, distribution, and reproduction in any medium, provided the original author and source are credited.

\section{Introduction}

Soft tissue fillers are designed to lift the tissue and add volume to improve the aesthetic appearance of wrinkles, folds, or volume lost during aging.

In 1981 the first bovine collagen filler received FDA approval, however this filler had the potential for allergic reactions. Since then, non-animal-based Hyaluronic Acid (HA), which had been used for intra-articular joint injection and ophthalmologic procedures for many years with a very good safety profile, was introduced and has become the most commonly used facial filler over the past several years [1]. HA fillers show excellent efficacy not only in correcting wrinkles but also in restoring tissue volume with minimal downtime.

These fillers are easy to use, allergy-free, and enzymatically degradable using an injection of hyaluronidase in case of a bad result. Thus, HA fillers have become a key tool in aesthetic surgery and medicine, and numerous products are commercially available. HA facial fillers usually lasts about 6-8 months, although longlasting effect have been described after deep HA injections, above the bones. The first ones describing a long lasting effect after deep facial injections of HA filler were Mashiko T, et al. injecting HA deeply, there is an injury and persisting inflammatory changes around the injected HA particles that are expected to activate periosteal stem cells and contribute to the induction of tissue neogenesis, such as formation of capsule, fibrosis, and calcification/ossification during the HA absorption process [2]. This new issue regarding long lasting effect of HA volumization can be especially useful in aesthetic improvements after major reconstructive surgical procedures of the face.

\section{Case Presentation}

In September 2018, at the Maxillo Facial Complex Unit of the University of Campania "Luigi Vanvitelli", I performed the reconstruction of an oral defect, following surgical resection of squamous cell carcinoma of the cheek, using a Superficial Temporal Artery Perforator (STAP) flap; STAP flap is a really useful tool for the reconstruction of various facial defects, however the main side effect of this procedure is represented by the donor site morbidity. The area where the flap is harvested can't be closed primary but need a skin graft. Placing a skin graft directly over the periosteum of the frontal area let to achieve a convex appearance of it (Figure 1).

Once surgical sites were completely healed, 3 months after surgery I approached the surgical sequela of the donor site with a non surgical approach, performing sub and supraperiosteal injections of a $20 \mathrm{mg} /$ mL crosslinked HA filler (Hyamira BASIC, NYUMA PHARM s.r.l., Arona, Italy). Injections were performed every 3 weeks, a total of 4 sessions were performed, and, on average, $0.4 \mathrm{~mL}$ of $\mathrm{HA}$ per session were used. During each session a big resistance was noted injecting HA, of course this was due to the scar tissue developed under the skin graft.

After each session a progressive volumization of the area was seen, moreover the skin graft, at the beginning completely attached and not mobile from the underlining frontal bone, showed a progressive improving in softness and mobility. Nine months after last injection the treated area showed a stable result, both in terms of volumization and softness (Figure 2).

\section{Discussion}

In the last 10 years, few papers have raised the attention to a most comprehensive aesthetic approach in facial restoration in case of congenital or acquired (trauma, tumor resection) anomalies with fillers [3].

Mashiko T, et al., were the first ones focusing about cross linked HA fillers used for major facial defects repair, such as Parry-Romberg syndrome, lupus erythematosus profundus, localized scleroderma, hemifacial microsomia, and posttraumatic or postsurgical craniomaxillofacial deformity reconstruction. The hypothesis is based on the concept that the volumizing effects result from tissue induction through activation of periosteum-resident stem cells. One clinical example, explaining our hypothesis, is cauliflower ear, a condition caracthreized by hypertrophied tissue developed after repeated traumatic hematoma in the auricle [4]. 


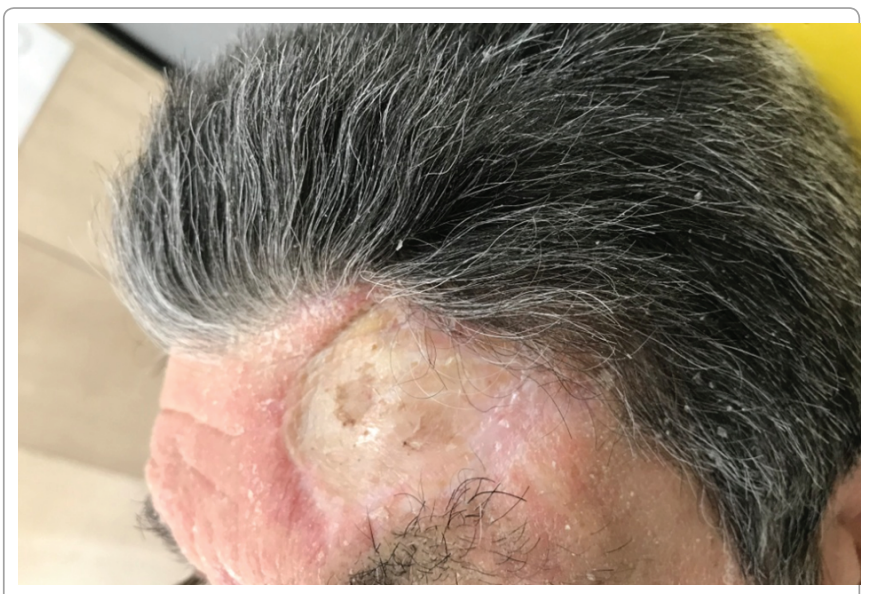

Figure 1: Donor site of STAP flap with the skin graft healed.

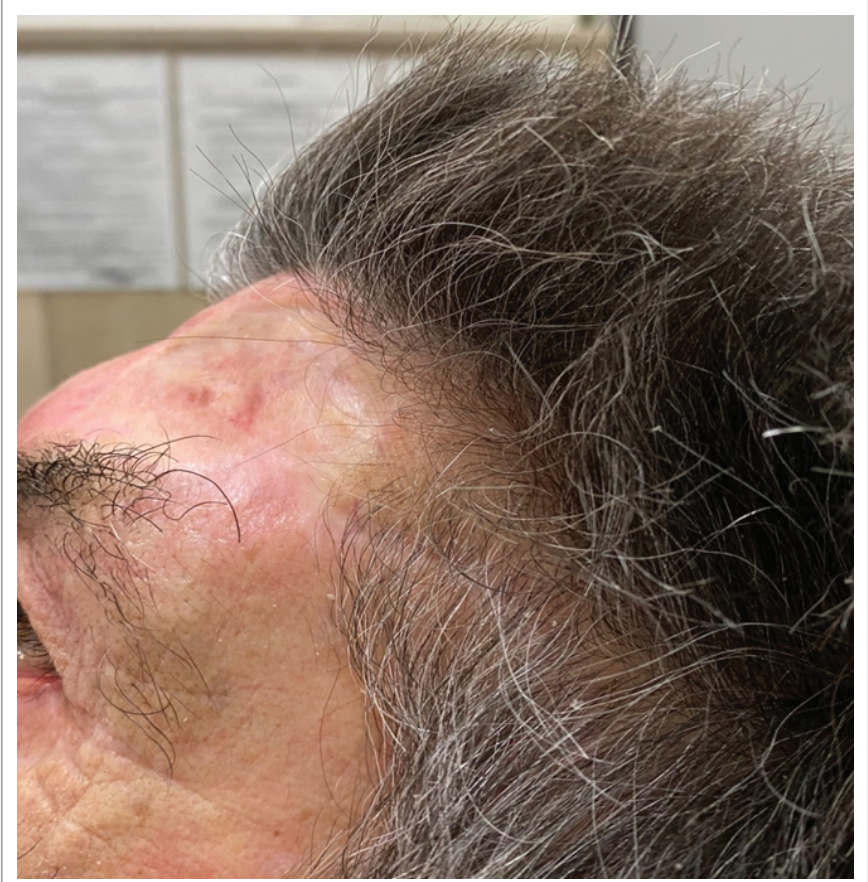

Figure 2: The same donor site after 9 months from HA injections.

The perichondrium has the potential for chondrogenesis, and after hematoma formation (perichondrium detachment from the cartilage), activated perichondrium-resident stem cells may fill the dead space with chondrogenesis, fibrosis, and/or ossification. Secondly, new bone formation at the edge of a tissue expander was experimentally demonstrated in rabbits, and this bone formation was induced by activated periosteal stem cells in the elevated periosteum $[5,6]$.

In the present paper we treated a surgical sequela characterized by a convex appearance of the skin after skin graft healing, we followed the hypothesis developed by Mashiko T, et al. performing multiple injection session of a $20 \mathrm{mg} / \mathrm{mL}$ crosslinked HA filler (Hyamira BASIC, NYUMA PHARM s.r.l., Arona, Italy) under the periosteum, inducing an injury and persisting inflammatory changes around the injected HA particles expecting to activate periosteal stem cells and contribute to induce tissue neogenesis, such as formation of capsule, fibrosis, and calcification/ossification during the HA absorption process; however, also injections over the periosteum were performed, in the fibrotic tissue lining under the skin graft.

Nine months after the injections, a stable more concave appearance of the defect was noted, the skin graft appeared also softener and more mobile compared to the pre injections time, probably these features were secondary to the improvement induced by HA injections into the fibrotic tissue between the bone and the skin graft.

\section{Conclusion}

Often and often, patients treated for complex facial reconstruction are not prone to have further surgeries to improve post surgical sequelas, however, can be much more prone to get improvements with non surgical approaches such as HA injections.

Not all HA fillers can be useful to face cases of congenital or acquired facial deformities; HA's physical properties are differents among different brands, studies are needed to understand the right filler to be used for each clinical conditions based on its own rheology.

Although, actually, HA fillers are mainly used for aesthetic purposes and only a few scientific papers point out their role in "post surgical filed", in a really next future, HA fillers could become an easy long lasting tool to improve post surgical facial deformities.

\section{References}

1. Goa KL, Benfield P (1994) Hyaluronic acid: A review of its pharmacology and use as a surgical aid in ophthalmology, and its therapeutic potential in joint disease and wound healing. Drugs 47: 536-566.

2. Mashiko T, Mori H, Kato H, Doi K, Kuno S, et al. (2013) Semipermanent volumization by an absorbable filler: onlay injection technique to the bone. Plast Reconstr Surg Glob Open 1: e4-e14.

3. Rauso R, Giudice GL, Zerbinati N, Tartaro G (2019) Hyaluronic Acid Injections as Nonsurgical Alternative in case of Delayed Diagnosis of Malar Arch Fracture: Case Report and Literature Review. Case Reports in Surgery 1360741.

4. Ohlsén L, Skoog T, Sohn SA (1975) The pathogenesis of cauliflower ear. An experimental study in rabbits. Scand J Plast Reconstr Surg 9: 34-39.

5. Benecke JE Jr, Gadre AK, Linthicum FH Jr (1990) Chondrogenic potential of tragal perichondrium: a cause of hearing loss following stapedectomy. Laryngoscope 100: 1292-1293.

6. Abrahamsson P, Isaksson S, Gordh M (2009) Periosteal expansion of rabbit mandible with an osmotic self-inflatable expander. Scand J Plast Reconstr Surg Hand Surg 43: 121-125. 